\title{
Single Step Optimal Block Matched Motion Estimation with Motion Vectors Having Arbitrary Pixel Precisions
}

\author{
C.Y.F. Ho ${ }^{1}$, Student Member of IEEE, B.W.K. Ling², Senior Member of IEEE, B.S. Giovanni², Z.W. Chi², \\ and W.C. Siu ${ }^{3}$, Senior Member of IEEE \\ ${ }^{1}$ School of Mathematical Sciences, Queen Mary, University of London, London, United Kingdom \\ 2 Department of Electronic Engineering, King’s College London, London, United Kingdom \\ ${ }^{3}$ Department of Electronic and Information Engineering, The Hong Kong Polytechnic University, Hong Kong, China \\ c.ho@qmul.ac.uk, wing-kuen.ling@kcl.ac.uk, saverio_giovanni.blasi@kcl.ac.uk, zhiwei.chi@kcl.ac.uk, and \\ enwcsiu@polyu.edu.hk
}

\begin{abstract}
This paper proposes a non-linear block matched motion model with motion vectors having arbitrary pixel precisions. The optimal motion vector which minimizes the mean square error is solved analytically in a single step. Our proposed algorithm can be regarded as a generalization of conventional half pixel search algorithms and quarter pixel search algorithms because our proposed algorithm could achieve motion vectors with arbitrary pixel precisions. Also, the computational effort of our proposed algorithm is lower than that of conventional quarter pixel search algorithms because our proposed algorithm could achieve motion vectors in a single step.
\end{abstract}

\section{INTRODUCTION}

Motion estimations play an important role in motion tracking applications, such as in a respiratory motion tracking application [1] and in a facial motion tracking application [2]. The most common motion estimation algorithm is the block matched motion estimation algorithm [3]. The current frame is usually partitioned into numbers of macro blocks with fixed or variable sizes. Each macro block in the current frame is compared with a number of macro blocks in the reference frame translated within a search window. Block matching errors are calculated based on a predefined cost function. The macro block in the reference frame that gives the minimum block matching error is considered as the best approximation of the macro block in the current frame. Each macro block in the current frame is represented by the best macro block in the reference frame, the motion vector (the motion vector is the vector representing the translation of the macro block in the reference frame.) and the residue (the residue is the difference between the macro block in the current frame and the best translated macro block in the reference frame).

The most common block matched motion estimation algorithm is the full integer pixel search algorithm. The full integer pixel search algorithm is a centre based algorithm in which all integer pixel locations in the search window are examined. However, the motion vectors are not necessarily represented by integer pixel precisions and a large portion of macro blocks in the current frame are best approximated by the macro blocks in the reference frame translated within a plus or a minus one pixel range around integer pixel locations. Hence, block matching errors could be further reduced if motion vectors are represented by non-integer pixel precisions. Conventional non-integer pixel search algorithms start searching pixels at half pixel locations. Half pixels are interpolated by nearby pixels at integer pixel locations. Block matching errors at some or all half pixel locations are evaluated. The half pixel location with the minimum block matching error is chosen. Similarly, quarter pixels are interpolated by nearby pixels at half pixel and integer pixel locations. The quarter pixel location with the minimum block matching error is chosen. Finer pixel locations could be evaluated successively. Since the block matching errors at finer pixel locations are evaluated via interpolations from the coarser pixel locations, if motion vectors with very fine pixel precisions are required, then many pixel locations are required to be evaluated. Hence, computational efforts of these algorithms are very heavy and these algorithms are very inefficient. Also, existing pixel search algorithms could only achieve motion vectors with rational pixel precisions. If the true motion vector is with an irrational pixel precision, then an infinite number of pixel locations have to be evaluated.

Interpolations are implemented via some predefined functions, such as a real valued quadratic function with two variables [4], a paraboloid function [5] and a straight line [6]. As the block matching error is a highly non-linear and non-convex function of the motion vector, it is very difficult to solve the motion vector that globally minimizes the block matching error. Hence, many pixel locations are still required to be evaluated and the pixel location with the lowest block matching error is chosen. Similar to conventional quarter pixel search algorithms, computational efforts of these algorithms are still very heavy and these algorithms are still very inefficient. Also, if the true motion vector is with an irrational pixel precision, then an infinite number of pixel locations still have to be evaluated.

In this paper, we propose a non-linear block matched motion model with motion vectors having arbitrary pixel precisions. The optimal motion vector which minimizes the mean square error is solved analytically in a single step. Our proposed algorithm has the following salient features. 1) The block matching error is evaluated in a single step which globally minimizes the mean square 
error. As the calculation of the mean square error at a fine pixel location is not derived from the coarser pixel locations, the computational effort of our proposed algorithm is much lower than that of conventional quarter pixel search algorithms. 2) Our proposed algorithm could achieve the true motion vector even though the true motion vector is with an irrational pixel precision. Computer numerical simulations show that the mean square errors of various video sequences based on our proposed algorithm are lower than that based on conventional half pixel search algorithms and quarter pixel search algorithms. The rest part of this paper is organized as follows. Section II describes our proposed non-linear block matched motion model. Section III derives analytically the optimal motion vector which minimizes the mean square error. Computer numerical simulations are presented in Section IV. Finally, a conclusion is drawn in Section V.

\section{Proposed Non-Linear Block Matched MOTION MODEL}

Denote the size of a macro block as $N \times N$, where $N \in Z^{+} . \forall k \in Z^{+}$, let $B_{k+1}$ be a macro block in the $k+1^{\text {th }}$ current frame and $B_{k+1}(x, y)$ be the pixel value of $B_{k+1}$ at the pixel location $(x, y) \quad \forall x \in\{0, \cdots, N-1\}$ and $\forall y \in\{0, \cdots, N-1\}$. Similarly, $\forall k \in Z^{+}$, let $B_{k}$ be a macro block in the $k^{\text {th }}$ reference frame and $B_{k}(x, y)$ be the pixel value of $B_{k}$ at the pixel location $(x, y) \forall x \in\{0, \cdots, N-1\}$ and $\forall y \in\{0, \cdots, N-1\} . \forall k \in Z^{+}$, denote the motion vector of $B_{k}$ as $\left(p_{0, k}+p_{k}, q_{0, k}+q_{k}\right)$, where $\left(p_{0, k}, q_{0, k}\right) \in Z^{2}$ and $\left(p_{k}, q_{k}\right) \in[-1,1] \times[-1,1] . \forall k \in Z^{+},\left(p_{0, k}, q_{0, k}\right)$ is the best integer pixel location which minimizes the block matching error and can be obtained via existing full integer pixel search algorithms. On the other hand, $\forall k \in Z^{+},\left(p_{k}, q_{k}\right)$ is the fine shift within $[-1,1] \times[-1,1]$ around $\left(p_{0, k}, q_{0, k}\right)$ and the values of $p_{k}$ and $q_{k}$ could be either rational or irrational. If the motion vector moves in different directions, then different pixels are required for interpolations. $\forall k \in Z^{+}$and $\forall\left(p_{k}, q_{k}\right) \in[0,1] \times[0,1]$, denote $\widetilde{B}_{k, p_{k}, q_{k}}^{U L}$ as the translated block of $B_{k}$ if the motion vector moves in the upper left direction, $\widetilde{B}_{k, p_{k}, q_{k}}^{U R}$ as the translated block of $B_{k}$ if the motion vector moves in the upper right direction, $\tilde{B}_{k, p_{k}, q_{k}}^{L L}$ as the translated block of $B_{k}$ if the motion vector moves in the lower left direction, and $\widetilde{B}_{k, p_{k}, q_{k}}^{L R}$ as the translated block of $B_{k}$ if the motion vector moves in the lower right direction. $\forall k \in Z^{+}$, $\forall\left(p_{k}, q_{k}\right) \in[0,1] \times[0,1] \quad, \quad \forall x \in\{0, \cdots, N-1\} \quad$ and $\forall y \in\{0, \cdots, N-1\}$, denote $\widetilde{B}_{k, p_{k}, q_{k}}^{U L}(x, y), \widetilde{B}_{k, p_{k}, q_{k}}^{U R}(x, y)$, $\widetilde{B}_{k, p_{k}, q_{k}}^{L L}(x, y)$ and $\widetilde{B}_{k, p_{k}, q_{k}}^{L R}(x, y)$ be the pixel values of $\widetilde{B}_{k, p_{k}, q_{k}}^{U L}, \widetilde{B}_{k, p_{k}, q_{k}}^{U R}, \widetilde{B}_{k, p_{k}, q_{k}}^{L L}$ and $\widetilde{B}_{k, p_{k}, q_{k}}^{L R}$ at the pixel location $(x, y)$, respectively. In this paper, $\forall k \in Z^{+}$, $\forall\left(p_{k}, q_{k}\right) \in[0,1] \times[0,1] \quad, \quad \forall x \in\{0, \cdots, N-1\} \quad$ and $\forall y \in\{0, \cdots, N-1\}, \widetilde{B}_{k, p_{k}, q_{k}}^{U L}(x, y), \widetilde{B}_{k, p_{k}, q_{k}}^{U R}(x, y), \widetilde{B}_{k, p_{k}, q_{k}}^{L L}(x, y)$ and $\widetilde{B}_{k, p_{k}, q_{k}}^{L R}(x, y)$ are constructed via the following models:

$$
\begin{aligned}
& \tilde{B}_{k, p_{k}, q_{k}}^{U L}(x, y) \equiv\left(1-p_{k}\right)\left(1-q_{k}\right) B_{k}\left(x+p_{0, k}, y+q_{0, k}\right) \\
& +\left(1-p_{k}\right) q_{k} B_{k}\left(x+p_{0, k}+1, y+q_{0, k}\right) \\
& +p_{k}\left(1-q_{k}\right) B_{k}\left(x+p_{0, k}, y+q_{0, k}+1\right) \\
& +p_{k} q_{k} B_{k}\left(x+p_{0, k}+1, y+q_{0, k}+1\right) \\
& \tilde{B}_{k, p_{k}, q_{k}}^{U R}(x, y) \equiv\left(1-p_{k}\right)\left(1-q_{k}\right) B_{k}\left(x+p_{0, k}, y+q_{0, k}\right) \\
& +\left(1-p_{k}\right) q_{k} B_{k}\left(x+p_{0, k}+1, y+q_{0, k}\right) \\
& +p_{k}\left(1-q_{k}\right) B_{k}\left(x+p_{0, k}, y+q_{0, k}-1\right) \\
& +p_{k} q_{k} B_{k}\left(x+p_{0, k}+1, y+q_{0, k}-1\right) \\
& \widetilde{B}_{k, p_{k}, q_{k}}^{L L}(x, y) \equiv\left(1-p_{k}\right)\left(1-q_{k}\right) B_{k}\left(x+p_{0, k}, y+q_{0, k}\right) \\
& +\left(1-p_{k}\right) q_{k} B_{k}\left(x+p_{0, k}-1, y+q_{0, k}\right) \\
& +p_{k}\left(1-q_{k}\right) B_{k}\left(x+p_{0, k}, y+q_{0, k}+1\right) \\
& +p_{k} q_{k} B_{k}\left(x+p_{0, k}-1, y+q_{0, k}+1\right)
\end{aligned}
$$

and

$$
\begin{aligned}
& \tilde{B}_{k, p_{k}, q_{k}}^{L R}(x, y) \equiv\left(1-p_{k}\right)\left(1-q_{k}\right) B_{k}\left(x+p_{0, k}, y+q_{0, k}\right) \\
& +\left(1-p_{k}\right) q_{k} B_{k}\left(x+p_{0, k}-1, y+q_{0, k}\right) \\
& +p_{k}\left(1-q_{k}\right) B_{k}\left(x+p_{0, k}, y+q_{0, k}-1\right) \\
& +p_{k} q_{k} B_{k}\left(x+p_{0, k}-1, y+q_{0, k}-1\right)
\end{aligned}
$$

respectively. $\forall k \in Z^{+}$and $\forall\left(p_{k}, q_{k}\right) \in[-1,1] \times[-1,1]$, let the mean square error between the translated $B_{k}$ and $B_{k+1}$ be $\operatorname{MSE}_{k}\left(p_{k}, q_{k}\right)$. That is, $\forall k \in Z^{+}$and $\forall\left(p_{k}, q_{k}\right) \in[-1,1] \times[-1,1]$,

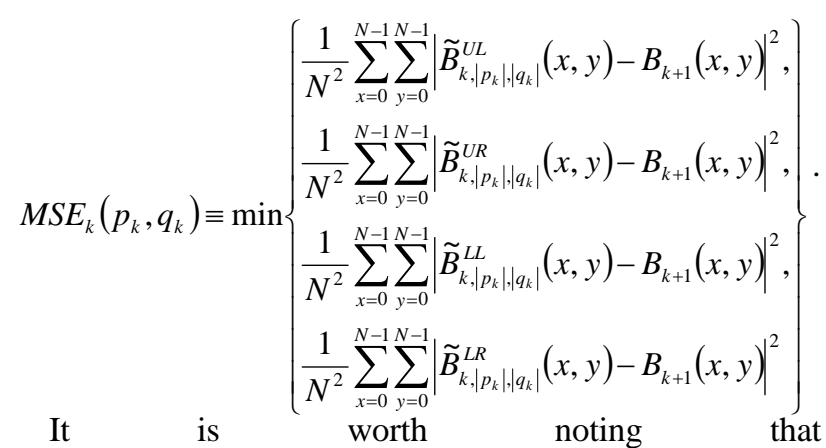
$\left(1-p_{k}\right)\left(1-q_{k}\right)+p_{k}\left(1-q_{k}\right)+\left(1-p_{k}\right) q_{k}+p_{k} q_{k}=1 \quad \forall k \in Z^{+}$ and $\forall\left(p_{k}, q_{k}\right) \in[0,1] \times[0,1]$. Hence, the average intensity of $\tilde{B}_{k, p_{k}, q_{k}}^{U L}, \widetilde{B}_{k, p_{k}, q_{k}}^{U R}, \widetilde{B}_{k, p_{k}, q_{k}}^{L L}$ and $\tilde{B}_{k, p_{k}, q_{k}}^{L R}$ will not be boosted up or attenuated down $\forall k \in Z^{+}$and $\forall\left(p_{k}, q_{k}\right) \in[0,1] \times[0,1]$.

If the true motion vector is located at the integer pixel locations, then it is obvious to see that $p_{k}=q_{k}=0$, or $p_{k}=0$ and $q_{k}=1$, or $p_{k}=1$ and $q_{k}=0$, or $p_{k}=q_{k}=1$. If the true motion vector is located at the half pixel locations, then it is obvious to see that $p_{k}=0$ and $q_{k}=\frac{1}{2}$, or $p_{k}=\frac{1}{2}$ and $q_{k}=0$, or $p_{k}=\frac{1}{2}$ and $q_{k}=1$, or $p_{k}=1$ and $q_{k}=\frac{1}{2}$, or $p_{k}=q_{k}=\frac{1}{2}$. If the true motion vector is located at the quarter pixel locations, then it is obvious to see that $p_{k}=q_{k}=\frac{1}{4}$, or $p_{k}=\frac{3}{4}$ and $q_{k}=\frac{1}{4}$, or $p_{k}=\frac{1}{4}$ and 
$q_{k}=\frac{3}{4}$, or $p_{k}=q_{k}=\frac{3}{4}$, or $p_{k}=0$ and $q_{k}=\frac{1}{4}$, or $p_{k}=0$ and $q_{k}=\frac{3}{4}$, or $p_{k}=1$ and $q_{k}=\frac{1}{4}$, or $p_{k}=1$ and $q_{k}=\frac{3}{4}$, or $p_{k}=\frac{1}{4}$ and $q_{k}=0$, or $p_{k}=\frac{3}{4}$ and $q_{k}=0$, or $p_{k}=\frac{1}{4}$ and $q_{k}=1$, or $p_{k}=\frac{3}{4}$ and $q_{k}=1$, or $p_{k}=\frac{1}{2}$ and $q_{k}=\frac{1}{4}$, or $p_{k}=\frac{1}{4}$ and $q_{k}=\frac{1}{2}$, or $p_{k}=\frac{1}{2}$ and $q_{k}=\frac{3}{4}$, or $p_{k}=\frac{3}{4}$ and $q_{k}=\frac{1}{2}$. Hence, integer pixel locations, half pixel locations and quarter pixel locations are particular locations represented by our proposed model.

\section{Derivation of Optimal Motion Vector}

The objective of the block matched motion estimation problem is to find $\left(p_{k}, q_{k}\right) \in[-1,1] \times[-1,1]$ such that $\operatorname{MSE}_{k}\left(p_{k}, q_{k}\right)$ is minimized $\forall k \in Z^{+} . \forall k \in Z^{+}$and $\forall\left(p_{k}, q_{k}\right) \in[0,1] \times[0,1]$, denote

$$
\begin{aligned}
& \operatorname{MSE}_{k}^{U L}\left(p_{k}, q_{k}\right) \\
& \left.\equiv \frac{1}{N^{2}} \sum_{x=0}^{N-1} \sum_{y=0}^{N-1}\left(\begin{array}{l}
\left(1-p_{k}\right)\left(1-q_{k}\right) B_{k}\left(x+p_{0, k}, y+q_{0, k}\right) \\
+\left(1-p_{k}\right) q_{k} B_{k}\left(x+p_{0, k}+1, y+q_{0, k}\right) \\
+p_{k}\left(1-q_{k}\right) B_{k}\left(x+p_{0, k}, y+q_{0, k}+1\right) \\
+p_{k} q_{k} B_{k}\left(x+p_{0, k}+1, y+q_{0, k}+1\right)-B_{k+1}(x, y)
\end{array}\right)\right)^{2},
\end{aligned}
$$

$\operatorname{MSE}_{k}^{U R}\left(p_{k}, q_{k}\right)$

$$
\equiv \frac{1}{N^{2}} \sum_{x=0}^{N-1} \sum_{y=0}^{N-1}\left(\begin{array}{l}
\left(1-p_{k}\right)\left(1-q_{k}\right) B_{k}\left(x+p_{0, k}, y+q_{0, k}\right) \\
+\left(1-p_{k}\right) q_{k} B_{k}\left(x+p_{0, k}+1, y+q_{0, k}\right) \\
+p_{k}\left(1-q_{k}\right) B_{k}\left(x+p_{0, k}, y+q_{0, k}-1\right) \\
+p_{k} q_{k} B_{k}\left(x+p_{0, k}+1, y+q_{0, k}-1\right)-B_{k+1}(x, y)
\end{array}\right)^{2},
$$

$\operatorname{MSE}_{k}^{L L}\left(p_{k}, q_{k}\right)$

$$
\equiv \frac{1}{N^{2}} \sum_{x=0}^{N-1} \sum_{y=0}^{N-1}\left(\begin{array}{l}
\left(1-p_{k}\right)\left(1-q_{k}\right) B_{k}\left(x+p_{0, k}, y+q_{0, k}\right) \\
+\left(1-p_{k}\right) q_{k} B_{k}\left(x+p_{0, k}-1, y+q_{0, k}\right) \\
+p_{k}\left(1-q_{k}\right) B_{k}\left(x+p_{0, k}, y+q_{0, k}+1\right) \\
+p_{k} q_{k} B_{k}\left(x+p_{0, k}-1, y+q_{0, k}+1\right)-B_{k+1}(x, y)
\end{array}\right)^{2}
$$

and

$$
\begin{aligned}
& \operatorname{MSE}_{k}^{L R}\left(p_{k}, q_{k}\right) \\
& \equiv \frac{1}{N^{2}} \sum_{x=0}^{N-1} \sum_{y=0}^{N-1}\left(\begin{array}{l}
\left(1-p_{k}\right)\left(1-q_{k}\right) B_{k}\left(x+p_{0, k}, y+q_{0, k}\right) \\
+\left(1-p_{k}\right) q_{k} B_{k}\left(x+p_{0, k}-1, y+q_{0, k}\right) \\
+p_{k}\left(1-q_{k}\right) B_{k}\left(x+p_{0, k}, y+q_{0, k}-1\right) \\
+p_{k} q_{k} B_{k}\left(x+p_{0, k}-1, y+q_{0, k}-1\right)-B_{k+1}(x, y)
\end{array}\right)^{2} .
\end{aligned}
$$

Then $\forall k \in Z^{+}$and $\forall\left(p_{k}, q_{k}\right) \in[0,1] \times[0,1]$, we have

$$
\begin{aligned}
& \operatorname{MSE}_{k}^{U L}\left(p_{k}, q_{k}\right) \\
& =\frac{1}{N^{2}} \sum_{x=0}^{N-1} \sum_{y=0}^{N-1}\left(\begin{array}{l}
p_{k} q_{k}\left(\begin{array}{l}
B_{k}\left(x+p_{0, k}, y+q_{0, k}\right) \\
-B_{k}\left(x+p_{0, k}, y+q_{0, k}+1\right) \\
-B_{k}\left(x+p_{0, k}+1, y+q_{0, k}\right) \\
+B_{k}\left(x+p_{0, k}+1, y+q_{0, k}+1\right)
\end{array}\right) \\
+p_{k}\left(\begin{array}{l}
B_{k}\left(x+p_{0, k}, y+q_{0, k}+1\right) \\
-B_{k}\left(x+p_{0, k}, y+q_{0, k}\right)
\end{array}\right) \\
+q_{k}\left(\begin{array}{l}
B_{k}\left(x+p_{0, k}+1, y+q_{0, k}\right) \\
-B_{k}\left(x+p_{0, k}, y+q_{0, k}\right)
\end{array}\right) \\
+B_{k}\left(x+p_{0, k}, y+q_{0, k}\right)-B_{k+1}(x, y)
\end{array}\right) .
\end{aligned}
$$

This further implies that $\forall k \in Z^{+}$and $\forall\left(p_{k}, q_{k}\right) \in[0,1] \times[0,1]$,

$$
\begin{aligned}
& \frac{\partial M S E_{k}^{U L}\left(p_{k}, q_{k}\right)}{\partial p_{k}}
\end{aligned}
$$

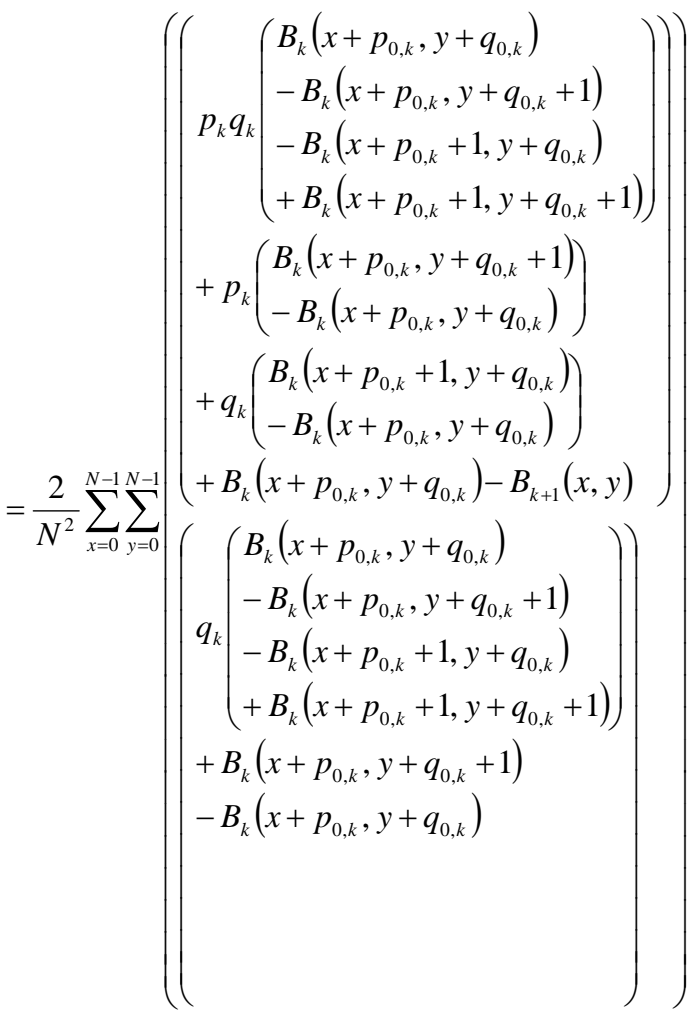

$$
\begin{aligned}
& =p_{k} q_{k}^{2} \sum_{x=0}^{N-1} \sum_{y=0}^{N-1} \frac{2}{N^{2}}\left(\begin{array}{l}
B_{k}\left(x+p_{0, k}, y+q_{0, k}\right) \\
-B_{k}\left(x+p_{0, k}, y+q_{0, k}+1\right) \\
-B_{k}\left(x+p_{0, k}+1, y+q_{0, k}\right) \\
+B_{k}\left(x+p_{0, k}+1, y+q_{0, k}+1\right)
\end{array}\right)^{2} \\
& +p_{k} q_{k} \sum_{x=0}^{N-1} \sum_{y=0}^{N-1} \frac{4}{N^{2}}\left(\begin{array}{l}
B_{k}\left(x+p_{0, k}, y+q_{0, k}\right) \\
-B_{k}\left(x+p_{0, k}, y+q_{0, k}+1\right) \\
-B_{k}\left(x+p_{0, k}+1, y+q_{0, k}\right) \\
+B_{k}\left(x+p_{0, k}+1, y+q_{0, k}+1\right)
\end{array}\right) \\
& \left(\begin{array}{l}
B_{k}\left(x+p_{0, k}, y+q_{0, k}+1\right) \\
-B_{k}\left(x+p_{0, k}, y+q_{0, k}\right)
\end{array}\right)
\end{aligned}
$$




$$
\begin{gathered}
+p_{k} \sum_{x=0}^{N-1} \sum_{y=0}^{N-1} \frac{2}{N^{2}}\left(\begin{array}{l}
B_{k}\left(x+p_{0, k}, y+q_{0, k}+1\right) \\
-B_{k}\left(x+p_{0, k}, y+q_{0, k}\right)
\end{array}\right) \\
\frac{2}{N^{2}}\left(\begin{array}{l}
B_{k}\left(x+p_{0, k}+1, y+q_{0, k}\right) \\
-B_{k}\left(x+p_{0, k}, y+q_{0, k}\right)
\end{array}\right) \\
+q_{k}^{2} \sum_{x=0}^{N-1} \sum_{y=0}^{N-1}\left(\begin{array}{l}
B_{k}\left(x+p_{0, k}, y+q_{0, k}\right) \\
-B_{k}\left(x+p_{0, k}, y+q_{0, k}+1\right) \\
-B_{k}\left(x+p_{0, k}+1, y+q_{0, k}\right) \\
+B_{k}\left(x+p_{0, k}+1, y+q_{0, k}+1\right)
\end{array}\right) \\
+\left(\begin{array}{l}
\left(\begin{array}{l}
B_{k}\left(x+p_{0, k}, y+q_{0, k}+1\right) \\
-B_{k}\left(x+p_{0, k}, y+q_{0, k}\right)
\end{array}\right) \\
\left(\begin{array}{l}
B_{k}\left(x+p_{0, k}+1, y+q_{0, k}\right) \\
-B_{k}\left(x+p_{0, k}, y+q_{0, k}\right)
\end{array}\right) \\
\left(\begin{array}{l}
B_{k}\left(x+p_{0, k}, y+q_{0, k}\right) \\
-B_{k}\left(x+p_{0, k}, y+q_{0, k}+1\right) \\
-B_{k}\left(x+p_{0, k}+1, y+q_{0, k}\right) \\
+B_{k}\left(x+p_{0, k}+1, y+q_{0, k}+1\right)
\end{array}\right) \\
+\sum_{x=0}^{N-1} \sum_{y=0}^{N-1} \frac{2}{N^{2}}\left(\begin{array}{l}
\left.B_{k}\left(x+p_{0, k}, y+q_{0, k}\right)-B_{k+1}(x, y)\right)
\end{array}\right)
\end{array}\right.
\end{gathered}
$$
$\forall k \in Z^{+}$, denote

$$
\begin{aligned}
& c_{k, p q^{2}} \equiv \sum_{x=0}^{N-1} \sum_{y=0}^{N-1} \frac{2}{N^{2}}\left(\begin{array}{l}
B_{k}\left(x+p_{0, k}, y+q_{0, k}\right) \\
-B_{k}\left(x+p_{0, k}, y+q_{0, k}+1\right) \\
-B_{k}\left(x+p_{0, k}+1, y+q_{0, k}\right) \\
+B_{k}\left(x+p_{0, k}+1, y+q_{0, k}+1\right)
\end{array}\right)^{2}, \\
& C_{k, p q} \equiv \sum_{x=0}^{N-1} \sum_{y=0}^{N-1} \frac{4}{N^{2}}\left(\begin{array}{l}
B_{k}\left(x+p_{0, k}, y+q_{0, k}\right) \\
-B_{k}\left(x+p_{0, k}, y+q_{0, k}+1\right) \\
-B_{k}\left(x+p_{0, k}+1, y+q_{0, k}\right) \\
+B_{k}\left(x+p_{0, k}+1, y+q_{0, k}+1\right)
\end{array}\right), \\
& \left(\begin{array}{l}
B_{k}\left(x+p_{0, k}, y+q_{0, k}+1\right) \\
-B_{k}\left(x+p_{0, k}, y+q_{0, k}\right)
\end{array}\right) \\
& C_{k, p} \equiv \sum_{x=0}^{N-1} \sum_{y=0}^{N-1} \frac{2}{N^{2}}\left(\begin{array}{l}
B_{k}\left(x+p_{0, k}, y+q_{0, k}+1\right) \\
-B_{k}\left(x+p_{0, k}, y+q_{0, k}\right)
\end{array}\right)^{2} \text {, } \\
& \frac{2}{N^{2}}\left(\begin{array}{l}
B_{k}\left(x+p_{0, k}+1, y+q_{0, k}\right) \\
-B_{k}\left(x+p_{0, k}, y+q_{0, k}\right)
\end{array}\right) \\
& c_{k, q^{2}} \equiv \sum_{x=0}^{N-1} \sum_{y=0}^{N-1}\left(\begin{array}{l}
B_{k}\left(x+p_{0, k}, y+q_{0, k}\right) \\
-B_{k}\left(x+p_{0, k}, y+q_{0, k}+1\right) \\
-B_{k}\left(x+p_{0, k}+1, y+q_{0, k}\right) \\
+B_{k}\left(x+p_{0, k}+1, y+q_{0, k}+1\right)
\end{array}\right),
\end{aligned}
$$

$$
c_{k, q} \equiv \sum_{x=0}^{N-1} \sum_{y=0}^{N-1} \frac{2}{N^{2}}\left(\begin{array}{l}
\left(\begin{array}{l}
B_{k}\left(x+p_{0, k}, y+q_{0, k}+1\right) \\
-B_{k}\left(x+p_{0, k}, y+q_{0, k}\right)
\end{array}\right) \\
\left(\begin{array}{l}
B_{k}\left(x+p_{0, k}+1, y+q_{0, k}\right) \\
-B_{k}\left(x+p_{0, k}, y+q_{0, k}\right)
\end{array}\right) \\
\left(\begin{array}{l}
B_{k}\left(x+p_{0, k}, y+q_{0, k}\right) \\
-B_{k}\left(x+p_{0, k}, y+q_{0, k}+1\right) \\
-B_{k}\left(x+p_{0, k}+1, y+q_{0, k}\right) \\
+B_{k}\left(x+p_{0, k}+1, y+q_{0, k}+1\right)
\end{array}\right) \\
\left(B_{k}\left(x+p_{0, k}, y+q_{0, k}\right)-B_{k+1}(x, y)\right)
\end{array}\right)
$$

and

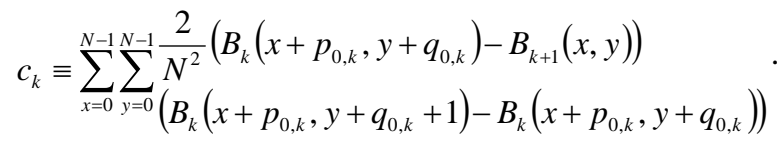

Then, $\forall k \in Z^{+}$and $\forall\left(p_{k}, q_{k}\right) \in[0,1] \times[0,1]$, we have

$$
\begin{aligned}
& \frac{\partial M S E_{k}^{U L}\left(p_{k}, q_{k}\right)}{\partial p_{k}} \\
& =p_{k}\left(c_{k, p q^{2}} q_{k}^{2}+c_{k, p q} q_{k}+c_{k, p}\right)+c_{k, q^{2}} q_{k}^{2}+c_{k, q} q_{k}+c_{k}
\end{aligned}
$$

Similarly, $\forall k \in Z^{+}$and $\forall\left(p_{k}, q_{k}\right) \in[0,1] \times[0,1]$,

$$
\frac{\partial M S E_{k}^{U L}\left(p_{k}, q_{k}\right)}{\partial q_{k}}
$$

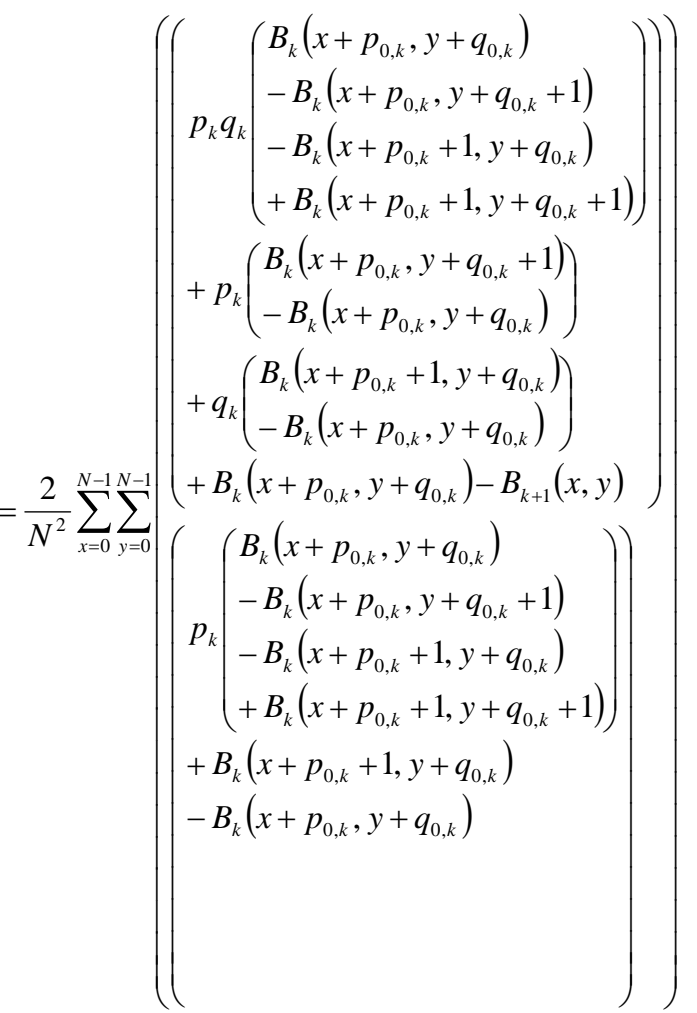

$$
\begin{aligned}
& =p_{k}^{2} q_{k} \sum_{x=0}^{N-1} \sum_{y=0}^{N-1} \frac{2}{N^{2}}\left(\begin{array}{l}
B_{k}\left(x+p_{0, k}, y+q_{0, k}\right) \\
-B_{k}\left(x+p_{0, k}, y+q_{0, k}+1\right) \\
-B_{k}\left(x+p_{0, k}+1, y+q_{0, k}\right) \\
+B_{k}\left(x+p_{0, k}+1, y+q_{0, k}+1\right)
\end{array}\right)^{2}
\end{aligned}
$$




$$
\begin{aligned}
& +p_{k} q_{k} \sum_{x=0}^{N-1} \sum_{y=0}^{N-1} \frac{4}{N^{2}}\left(\begin{array}{l}
B_{k}\left(x+p_{0, k}, y+q_{0, k}\right) \\
-B_{k}\left(x+p_{0, k}, y+q_{0, k}+1\right) \\
-B_{k}\left(x+p_{0, k}+1, y+q_{0, k}\right) \\
+B_{k}\left(x+p_{0, k}+1, y+q_{0, k}+1\right)
\end{array}\right) \\
& \left(\begin{array}{l}
B_{k}\left(x+p_{0, k}+1, y+q_{0, k}\right) \\
-B_{k}\left(x+p_{0, k}, y+q_{0, k}\right)
\end{array}\right) \\
& +q_{k} \sum_{x=0}^{N-1} \sum_{y=0}^{N-1} \frac{2}{N^{2}}\left(\begin{array}{l}
B_{k}\left(x+p_{0, k}+1, y+q_{0, k}\right) \\
-B_{k}\left(x+p_{0, k}, y+q_{0, k}\right)
\end{array}\right)^{2} \\
& \frac{2}{N^{2}}\left(\begin{array}{l}
B_{k}\left(x+p_{0, k}, y+q_{0, k}+1\right) \\
-B_{k}\left(x+p_{0, k}, y+q_{0, k}\right)
\end{array}\right) \\
& +p_{k}^{2} \sum_{x=0}^{N-1} \sum_{y=0}^{N-1}\left(\begin{array}{l}
B_{k}\left(x+p_{0, k}, y+q_{0, k}\right) \\
-B_{k}\left(x+p_{0, k}, y+q_{0, k}+1\right)
\end{array}\right. \\
& \left.\begin{array}{l}
-B_{k}\left(x+p_{0, k}+1, y+q_{0, k}\right) \\
+B_{k}\left(x+p_{0, k}+1, y+q_{0, k}+1\right)
\end{array}\right) \\
& \left(\left(\begin{array}{l}
B_{k}\left(x+p_{0, k}, y+q_{0, k}+1\right) \\
-B_{k}\left(x+p_{0, k}, y+q_{0, k}\right)
\end{array}\right)\right. \\
& \sum_{k-1 N-1}^{N-1}\left(\begin{array}{l}
B_{k}\left(x+p_{0, k}+1, y+q_{0, k}\right) \\
-B_{k}\left(x+p_{0, k}, y+q_{0, k}\right)
\end{array}\right)
\end{aligned}
$$

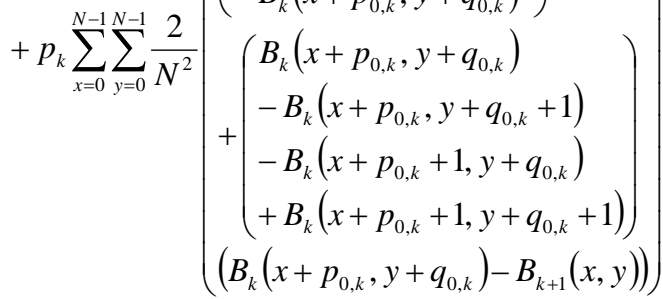

$$
\begin{aligned}
& \begin{array}{l}
+\sum_{x=0}^{N-1} \sum_{y=0}^{N-1} \frac{2}{N^{2}}\left(B_{k}\left(x+p_{0, k}+1, y+q_{0, k}\right)-B_{k}\left(x+p_{0, k}, y+q_{0, k}\right)\right) \\
.
\end{array}
\end{aligned}
$$

$\forall k \in Z^{+}$, denote

$$
\begin{aligned}
& z_{k, q p^{2}} \equiv \sum_{x=0}^{N-1} \sum_{y=0}^{N-1} \frac{2}{N^{2}}\left(\begin{array}{l}
B_{k}\left(x+p_{0, k}, y+q_{0, k}\right) \\
-B_{k}\left(x+p_{0, k}, y+q_{0, k}+1\right) \\
-B_{k}\left(x+p_{0, k}+1, y+q_{0, k}\right) \\
+B_{k}\left(x+p_{0, k}+1, y+q_{0, k}+1\right)
\end{array}\right)^{2}, \\
& z_{k, q p} \equiv \sum_{x=0}^{N-1} \sum_{y=0}^{N-1} \frac{4}{N^{2}}\left(\begin{array}{l}
B_{k}\left(x+p_{0, k}, y+q_{0, k}\right) \\
-B_{k}\left(x+p_{0, k}, y+q_{0, k}+1\right) \\
-B_{k}\left(x+p_{0, k}+1, y+q_{0, k}\right) \\
+B_{k}\left(x+p_{0, k}+1, y+q_{0, k}+1\right)
\end{array}\right), \\
& \left(\begin{array}{l}
B_{k}\left(x+p_{0, k}+1, y+q_{0, k}\right) \\
-B_{k}\left(x+p_{0, k}, y+q_{0, k}\right)
\end{array}\right) \\
& z_{k, q} \equiv \sum_{x=0}^{N-1} \sum_{y=0}^{N-1} \frac{2}{N^{2}}\left(\begin{array}{l}
B_{k}\left(x+p_{0, k}+1, y+q_{0, k}\right) \\
-B_{k}\left(x+p_{0, k}, y+q_{0, k}\right)
\end{array}\right)^{2},
\end{aligned}
$$

$$
\begin{gathered}
\frac{2}{N^{2}}\left(\begin{array}{l}
B_{k}\left(x+p_{0, k}, y+q_{0, k}+1\right) \\
-B_{k}\left(x+p_{0, k}, y+q_{0, k}\right)
\end{array}\right) \\
z_{k, p^{2}} \equiv \sum_{x=0}^{N-1} \sum_{y=0}^{N-1}\left(\begin{array}{l}
B_{k}\left(x+p_{0, k}, y+q_{0, k}\right) \\
-B_{k}\left(x+p_{0, k}, y+q_{0, k}+1\right) \\
-B_{k}\left(x+p_{0, k}+1, y+q_{0, k}\right) \\
+B_{k}\left(x+p_{0, k}+1, y+q_{0, k}+1\right)
\end{array}\right), \\
z_{k, p} \equiv \sum_{x=0}^{N-1} \sum_{y=0}^{N-1} \frac{2}{N^{2}}\left(\begin{array}{l}
\left(\begin{array}{l}
B_{k}\left(x+p_{0, k}, y+q_{0, k}+1\right) \\
-B_{k}\left(x+p_{0, k}, y+q_{0, k}\right)
\end{array}\right) \\
\left(\begin{array}{l}
B_{k}\left(x+p_{0, k}+1, y+q_{0, k}\right) \\
-B_{k}\left(x+p_{0, k}, y+q_{0, k}\right)
\end{array}\right) \\
\left(\begin{array}{l}
B_{k}\left(x+p_{0, k}, y+q_{0, k}\right) \\
-B_{k}\left(x+p_{0, k}, y+q_{0, k}+1\right) \\
-B_{k}\left(x+p_{0, k}+1, y+q_{0, k}\right) \\
+B_{k}\left(x+p_{0, k}+1, y+q_{0, k}+1\right)
\end{array}\right) \\
\left(B_{k}\left(x+p_{0, k}, y+q_{0, k}\right)-B_{k+1}(x, y)\right)
\end{array}\right)
\end{gathered}
$$

and

$$
z_{k} \equiv \sum_{x=0}^{N-1} \sum_{y=0}^{N-1} \frac{2}{N^{2}}\left(B_{k}\left(x+p_{0, k}+1, y+q_{0, k}\right)-B_{k}\left(x+p_{0, k}, y+q_{0, k}\right)\right) .
$$

Then $\forall k \in Z^{+}$and $\forall\left(p_{k}, q_{k}\right) \in[0,1] \times[0,1]$, we have

$$
\begin{aligned}
& \frac{\partial M S E_{k}^{U L}\left(p_{k}, q_{k}\right)}{\partial q_{k}} \\
& =q_{k}\left(z_{k, q p^{2}} p_{k}^{2}+z_{k, q p} p_{k}+z_{k, q}\right)+z_{k, p^{2}} p_{k}^{2}+z_{k, p} p_{k}+z_{k}
\end{aligned}
$$

$\forall k \in Z^{+}$, denote a stationary point of $\operatorname{MSE}_{k}^{U L}\left(p_{k}, q_{k}\right)$ as $\left(p_{k}^{U L *}, q_{k}^{U L *}\right)$. Then, $\forall k \in Z^{+}$, we have

$$
\left.\frac{\partial M S E_{k}^{U L}\left(p_{k}, q_{k}\right)}{\partial p_{k}}\right|_{\left(p_{k}, q_{k}\right)=\left(p_{k}^{U L *}, q_{k}^{U L *}\right)}=0
$$

and

$$
\left.\frac{\partial M S E_{k}^{U L}\left(p_{k}, q_{k}\right)}{\partial q_{k}}\right|_{\left(p_{k}, q_{k}\right)=\left(p_{k}^{U L *}, q_{k}^{U L *}\right)}=0 .
$$

If $\exists k \in Z^{+}$such that $c_{k, p q^{2}} \neq 0$ or $c_{k, p q} \neq 0$ or $c_{k, p} \neq 0$, then we have $p_{k}^{U L *}=-\frac{c_{k, q^{2}} q_{k}^{U L *^{2}}+c_{k, q} q_{k}^{U L^{* *}}+c_{k}}{c_{k, p q^{2}} q_{k}^{U L *^{2}}+c_{k, p q} q_{k}^{U L *}+c_{k, p}}$ and

$$
\begin{aligned}
& q_{k}^{U L L^{*}}\left(\begin{array}{c}
z_{k, q p^{2}}\left(-\frac{c_{k, q^{2}} q_{k}^{U L *^{2}}+c_{k, q} q_{k}^{U L *}+c_{k}}{c_{k, p q^{2}} q_{k}^{U L *^{2}}+c_{k, p q} q_{k}^{U L *}+c_{k, p}}\right)^{2} \\
+z_{k, q p}\left(-\frac{c_{k, q^{2}} q_{k}^{U L *^{2}}+c_{k, q} q_{k}^{U L *}+c_{k}}{c_{k, p q^{2}} q_{k}^{U L *^{2}}+c_{k, p q} q_{k}^{U L *}+c_{k, p}}\right)+z_{k, q}
\end{array}\right) \\
& +z_{k, p^{2}}\left(-\frac{c_{k, q^{2}} q_{k}^{U L *^{2}}+c_{k, q} q_{k}^{U L *}+c_{k}}{c_{k, p q^{2}} q_{k}^{U *^{2}}+c_{k, p q} q_{k}^{U L *}+c_{k, p}}\right)^{2} \\
& +z_{k, p}\left(-\frac{c_{k, q^{2}} q_{k}^{U L *^{2}}+c_{k, q} q_{k}^{U L *}+c_{k}}{c_{k, p q^{2}} q_{k}^{U L * *^{2}}+c_{k, p q} q_{k}^{U L *}+c_{k, p}}\right)+z_{k}=0
\end{aligned}
$$


which further implies that

$$
\begin{aligned}
& q_{k}^{U L *}\left(\begin{array}{l}
z_{k, q p^{2}}\left(c_{k, q^{2}} q_{k}^{U L *^{2}}+c_{k, q} q_{k}^{U L *}+c_{k}\right)^{2} \\
-z_{k, q p}\left(c_{k, q^{2}} q_{k}^{U L *^{2}}+c_{k, q} q_{k}^{U L *}+c_{k}\right) \\
\left(c_{k, p q^{2}} q_{k}^{U L^{2}}+c_{k, p q} q_{k}^{U L *}+c_{k, p}\right) \\
+z_{k, q}\left(c_{k, p q^{2}} q_{k}^{U L *^{2}}+c_{k, p q} q_{k}^{U L *}+c_{k, p}\right)^{2}
\end{array}\right) \\
& +Z_{k, p^{2}}\left(c_{k, q^{2}} q_{k}^{U L *^{2}}+c_{k, q} q_{k}^{U L *}+c_{k}\right)^{2} \\
& -Z_{k, p}\left(c_{k, q^{2}} q_{k}^{U L *^{2}}+c_{k, q} q_{k}^{U L *}+c_{k}\right) \\
& \left(c_{k, p q^{2}} q_{k}^{U L *^{2}}+c_{k, p q} q_{k}^{U L *}+c_{k, p}\right) \\
& +z_{k}\left(c_{k, p q^{2}} q_{k}^{U L *^{2}}+c_{k, p q} q_{k}^{U L *}+c_{k, p}\right)^{2}=0
\end{aligned}
$$

If $\exists k \in Z^{+}$such that $c_{k, p q^{2}}=0$ and $c_{k, p q}=0$ and $c_{k, p}=0$, but $z_{k, q p^{2}} \neq 0$ or $z_{k, q p} \neq 0$ or $z_{k, q} \neq 0$, then we have

$$
\begin{aligned}
q_{k}^{U L *}= & -\frac{z_{k, p^{2}} p_{k}^{U L *^{2}}+z_{k, p} p_{k}^{U L *}+z_{k}}{z_{k, q p^{2}} p_{k}^{U L *^{2}}+z_{k, q p} p_{k}^{U L *}+z_{k, q}} \text { and } \\
& c_{k, q^{2}}\left(-\frac{z_{k, p^{2}} p_{k}^{U L *^{2}}+z_{k, p} p_{k}^{U L *}+z_{k}}{z_{k, q p^{2}} p_{k}^{U L *^{2}}+z_{k, q p} p_{k}^{U L *}+z_{k, q}}\right)^{2}, \\
& +c_{k, q}\left(-\frac{z_{k, p^{2}} p_{k}^{U L *^{2}}+z_{k, p} p_{k}^{U L *}+z_{k}}{z_{k, q p^{2}} p_{k}^{U L *^{2}}+z_{k, q p} p_{k}^{U L *}+z_{k, q}}\right)+c_{k}=0
\end{aligned}
$$

which further implies that

$$
\begin{aligned}
& c_{k, q^{2}}\left(z_{k, p^{2}} p_{k}^{U L *^{2}}+z_{k, p} p_{k}^{U L *}+z_{k}\right)^{2} \\
& -c_{k, q}\left(z_{k, p^{2}} p_{k}^{U L *^{2}}+z_{k, p} p_{k}^{U L *}+z_{k}\right) \\
& \left(z_{k, q p^{2}} p_{k}^{U L *^{2}}+z_{k, q p} p_{k}^{U L *}+z_{k, q}\right) \\
& +c_{k}\left(z_{k, q p^{2}} p_{k}^{U L *^{2}}+z_{k, q p} p_{k}^{U L *}+z_{k, q}\right)^{2}=0
\end{aligned}
$$

If $\exists k \in Z^{+}$such that $c_{k, p q^{2}}=0$ and $c_{k, p q}=0$ and $c_{k, p}=0$ and $z_{k, q p^{2}}=0$ and $z_{k, q p}=0$ and $z_{k, q}=0$, then we have

$$
c_{k, q^{2}} q_{k}^{U L *^{2}}+c_{k, q} q_{k}^{U L *}+c_{k}=0
$$

and

$$
z_{k, p^{2}} p_{k}^{U L *^{2}}+z_{k, p} p_{k}^{U L *}+z_{k}=0 .
$$

By solving (1) or (2) or (3), $\left(p_{k}^{U L *}, q_{k}^{U L *}\right)$ can be found $\forall k \in Z^{+} . \forall k \in Z^{+}$, denote the total number of vectors $\left(p_{k}^{U L *}, q_{k}^{U L *}\right)$ within $[0,1] \times[0,1]$ as $M_{k}^{U L} . \forall k \in Z^{+}$, if $M_{k}^{U L} \geq 1$, then denote those vectors as $\left(p_{k, m}^{U L *}, q_{k, m}^{U L *}\right)$ for $m=1,2, \cdots, M_{k}^{U L} \quad$ and denote $\quad F_{k}^{U L} \equiv\left\{\left(p_{k, m}^{U L *}, q_{k, m}^{U L *}\right) \quad\right.$ for $\left.m=1,2, \cdots, M_{k}^{U L}\right\} \bigcup\{(0,0)\}$.

However, in general it is not guaranteed that $M_{k}^{U L} \geq 1$ $\forall k \in Z^{+}$. If $\exists k \in Z^{+}$such that $M_{k}^{U L}=0$, then there may be no stationary point or the stationary points are not in the feasible region $[0,1] \times[0,1]$. For these two cases, the global minimum of the $\operatorname{MSE}_{k}^{U L}\left(p_{k}, q_{k}\right)$ could be on the boundaries of the feasible region. Hence, it is required to check if there exist some stationary points on the boundaries of the feasible region. The following procedures are employed for the checking. $\forall k \in Z^{+}$and $\forall q_{k} \in[0,1]$,

$$
\begin{aligned}
& \operatorname{MSE}_{k}^{U L}\left(0, q_{k}\right) \\
& =\left.\frac{1}{N^{2}} \sum_{x=0}^{N-1} \sum_{y=0}^{N-1}\left(\begin{array}{l}
\left(1-p_{k}\right)\left(1-q_{k}\right) B_{k}\left(x+p_{0, k}, y+q_{0, k}\right) \\
+\left(1-p_{k}\right) q_{k} B_{k}\left(x+p_{0, k}+1, y+q_{0, k}\right) \\
+p_{k}\left(1-q_{k}\right) B_{k}\left(x+p_{0, k}, y+q_{0, k}+1\right) \\
+p_{k} q_{k} B_{k}\left(x+p_{0, k}+1, y+q_{0, k}+1\right) \\
-B_{k+1}(x, y)
\end{array}\right)\right|_{p_{k}=0} ^{2} . \\
& =\frac{1}{N^{2}} \sum_{x=0}^{N-1} \sum_{y=0}^{N-1}\left(\begin{array}{l}
\left(1-q_{k}\right) B_{k}\left(x+p_{0, k}, y+q_{0, k}\right) \\
+q_{k} B_{k}\left(x+p_{0, k}+1, y+q_{0, k}\right)-B_{k+1}(x, y)
\end{array}\right)^{2} \\
& =\frac{1}{N^{2}} \sum_{x=0}^{N-1} \sum_{y=0}^{N-1}\left(\begin{array}{l}
B_{k}\left(x+p_{0, k}+1, y+q_{0, k}\right) \\
q_{k}\left(\begin{array}{l}
B_{k}\left(x+p_{0, k}, y+q_{0, k}\right) \\
-B_{k}\left(x+p_{0, k}, y+q_{0, k}\right)-B_{k+1}(x, y)
\end{array}\right)
\end{array}\right)^{2}
\end{aligned}
$$

This implies that $\forall k \in Z^{+}$and $\forall q_{k} \in[0,1]$,

$$
\left.\begin{array}{c}
\frac{\partial M S E_{k}^{U L}\left(0, q_{k}\right)}{\partial q_{k}} \\
=\frac{2}{N^{2}} \sum_{x=0}^{N-1} \sum_{y=0}^{N-1}\left(\begin{array}{l}
q_{k}\left(\begin{array}{l}
B_{k}\left(x+p_{0, k}+1, y+q_{0, k}\right) \\
-B_{k}\left(x+p_{0, k}, y+q_{0, k}\right)
\end{array}\right) \\
+B_{k}\left(x+p_{0, k}, y+q_{0, k}\right)-B_{k+1}(x, y)
\end{array}\right) \\
\left(\begin{array}{l}
B_{k}\left(x+p_{0, k}+1, y+q_{0, k}\right) \\
-B_{k}\left(x+p_{0, k}, y+q_{0, k}\right)
\end{array}\right) \\
=\frac{2}{N^{2}} \sum_{x=0}^{N-1} \sum_{y=0}^{N-1}+\left(\begin{array}{l}
B_{k}\left(x+p_{0, k}+1, y+q_{0, k}\right) \\
-B_{k}\left(x+p_{0, k}, y+q_{0, k}\right)
\end{array}\right)
\end{array}\right) .
$$

$\forall k \in Z^{+}$, denote

$$
\tilde{c}_{k, 0, q} \equiv \frac{2}{N^{2}} \sum_{x=0}^{N-1} \sum_{y=0}^{N-1}\left(\begin{array}{l}
B_{k}\left(x+p_{0, k}+1, y+q_{0, k}\right) \\
-B_{k}\left(x+p_{0, k}, y+q_{0, k}\right)
\end{array}\right)^{2}
$$

and

$$
\widetilde{c}_{k, 0} \equiv \frac{2}{N^{2}} \sum_{x=0}^{N-1} \sum_{y=0}^{N-1}\left(\begin{array}{l}
\left.B_{k}\left(x+p_{0, k}, y+q_{0, k}\right)-B_{k+1}(x, y)\right) \\
-B_{k}\left(x+p_{0, k}+1, y+q_{0, k}\right)
\end{array}\right),
$$

then $\forall k \in Z^{+}$and $\forall q_{k} \in[0,1]$ we have

$$
\frac{\partial M S E_{k}^{U L}\left(0, q_{k}\right)}{\partial q_{k}}=\tilde{c}_{k, 0, q} q_{k}+\tilde{c}_{k, 0} .
$$

$\forall k \in Z^{+}$, denote a stationary point of $\operatorname{MSE}_{k}^{U L}\left(0, q_{k}\right)$ as $\left(0, \widetilde{q}_{k}^{0, U L}\right)$. If $\exists k \in Z^{+}$such that $\tilde{c}_{k, 0, q} \neq 0$, then 
$\left.\frac{\partial M S E_{k}^{U L}\left(0, q_{k}\right)}{\partial q_{k}}\right|_{q_{k}=\widetilde{q}_{k}^{0, U L}}=0$ implies that $\tilde{q}_{k}^{0, U L}=-\frac{\widetilde{c}_{k, 0}}{\widetilde{c}_{k, 0, q}} \cdot$ If this value is in the feasible region, that is $\exists k \in Z^{+}$such that $\widetilde{c}_{k, 0, q} \neq 0$ and $-\frac{\widetilde{c}_{k, 0}}{\widetilde{c}_{k, 0, q}} \in[0,1]$, then this stationary point could be the global minimum. For this case, define $\widetilde{F}_{k, 0, q}^{U L} \equiv\left\{\left(0,-\frac{\widetilde{c}_{k, 0}}{\widetilde{c}_{k, 0, q}}\right)\right\}$. However, the following three cases could be happened. (Case i) This stationary point may be outside the feasible region, that is $\exists k \in Z^{+}$such that $\tilde{c}_{k, 0, q} \neq 0$ and $-\frac{\widetilde{c}_{k, 0}}{\widetilde{c}_{k, 0, q}} \notin[0,1]$. (Case ii) $\exists k \in Z^{+}$such that $\widetilde{c}_{k, 0, q}=0$ and $\tilde{c}_{k, 0}=0$. Then, $\exists k \in Z^{+}$such that all the points on the boundary $p_{k}=0 \forall q_{k} \in[0,1]$ are stationary points, that is $\exists k \in Z^{+}$such that $\frac{\partial M S E_{k}^{U L}\left(0, q_{k}\right)}{\partial q_{k}}=0$ $\forall q_{k} \in[0,1]$. (Case iii) $\exists k \in Z^{+}$such that $\widetilde{c}_{k, 0, q}=0$ and $\tilde{c}_{k, 0} \neq 0$. Then, $\exists k \in Z^{+}$such that there is no stationary point on the boundary $p_{k}=0 \forall q_{k} \in[0,1]$, that is $\exists k \in Z^{+}$ such that $\frac{\partial M S E_{k}^{U L}\left(0, q_{k}\right)}{\partial q_{k}} \neq 0 \forall q_{k} \in[0,1]$. For all these three cases, we do not consider that the global minimum is on the boundary $p_{k}=0 \forall q_{k} \in(0,1]$. For these three cases, define $\widetilde{F}_{k, 0, q}^{U L} \equiv \phi$, where $\phi$ is denoted as the empty set. Similarly, $\forall k \in Z^{+}$and $\forall q_{k} \in[0,1]$,

$$
\begin{aligned}
& \operatorname{MSE}_{k}^{U L}\left(1, q_{k}\right) \\
& =\left.\frac{1}{N^{2}} \sum_{x=0}^{N-1} \sum_{y=0}^{N-1}\left(\begin{array}{l}
\left(1-p_{k}\right)\left(1-q_{k}\right) B_{k}\left(x+p_{0, k}, y+q_{0, k}\right) \\
+\left(1-p_{k}\right) q_{k} B_{k}\left(x+p_{0, k}+1, y+q_{0, k}\right) \\
+p_{k}\left(1-q_{k}\right) B_{k}\left(x+p_{0, k}, y+q_{0, k}+1\right) \\
+p_{k} q_{k} B_{k}\left(x+p_{0, k}+1, y+q_{0, k}+1\right) \\
-B_{k+1}(x, y)
\end{array}\right)\right|_{p_{k}=1} ^{2} \\
& =\frac{1}{N^{2}} \sum_{x=0}^{N-1} \sum_{y=0}^{N-1}\left(\begin{array}{l}
\left(1-q_{k}\right) B_{k}\left(x+p_{0, k}, y+q_{0, k}+1\right) \\
+q_{k} B_{k}\left(x+p_{0, k}+1, y+q_{0, k}+1\right)-B_{k+1}(x, y)
\end{array}\right)^{2} \\
& =\frac{1}{N^{2}} \sum_{x=0}^{N-1} \sum_{y=0}^{N-1}\left(\begin{array}{l}
B_{k}\left(x+p_{0, k}+1, y+q_{0, k}+1\right) \\
q_{k}\left(\begin{array}{l}
B_{k}\left(x+p_{0, k}, y+q_{0, k}+1\right) \\
+B_{k}\left(x+p_{0, k}, y+q_{0, k}+1\right)-B_{k+1}(x, y)
\end{array}\right)^{2}
\end{array}\right.
\end{aligned}
$$

This implies that $\forall k \in Z^{+}$and $\forall q_{k} \in[0,1]$,

$$
\begin{aligned}
& \frac{\partial M S E_{k}^{U L}\left(1, q_{k}\right)}{\partial q_{k}} \\
& =\frac{2}{N^{2}} \sum_{x=0}^{N-1} \sum_{y=0}^{N-1}\left(\begin{array}{l}
\left(\begin{array}{l}
B_{k}\left(x+p_{0, k}+1, y+q_{0, k}+1\right) \\
q_{k}\left(\begin{array}{l}
B_{k}\left(x+p_{0, k}, y+q_{0, k}+1\right)
\end{array}\right) \\
+B_{k}\left(x+p_{0, k}, y+q_{0, k}+1\right)-B_{k+1}(x, y)
\end{array}\right) \\
\left(\begin{array}{l}
B_{k}\left(x+p_{0, k}+1, y+q_{0, k}+1\right) \\
-B_{k}\left(x+p_{0, k}, y+q_{0, k}+1\right)
\end{array}\right)
\end{array}\right.
\end{aligned}
$$

$$
\begin{gathered}
q_{k}\left(\begin{array}{l}
B_{k}\left(x+p_{0, k}+1, y+q_{0, k}+1\right) \\
-B_{k}\left(x+p_{0, k}, y+q_{0, k}+1\right)
\end{array}\right)^{2} \\
=\frac{2}{N^{2}} \sum_{x=0}^{N-1} \sum_{y=0}^{N-1}+\left(\begin{array}{l}
\left.B_{k}\left(x+p_{0, k}, y+q_{0, k}+1\right)-B_{k+1}(x, y)\right) \\
\left(\begin{array}{l}
B_{k}\left(x+p_{0, k}+1, y+q_{0, k}+1\right) \\
-B_{k}\left(x+p_{0, k}, y+q_{0, k}+1\right)
\end{array}\right)
\end{array} .\right.
\end{gathered}
$$

$\forall k \in Z^{+}$, denote

$$
\widetilde{c}_{k, 1, q} \equiv \frac{2}{N^{2}} \sum_{x=0}^{N-1} \sum_{y=0}^{N-1}\left(\begin{array}{l}
B_{k}\left(x+p_{0, k}+1, y+q_{0, k}+1\right) \\
-B_{k}\left(x+p_{0, k}, y+q_{0, k}+1\right)
\end{array}\right)^{2}
$$

and

$$
\widetilde{c}_{k, 1} \equiv \frac{2}{N^{2}} \sum_{x=0}^{N-1} \sum_{y=0}^{N-1}\left(\begin{array}{l}
\left(B_{k}\left(x+p_{0, k}, y+q_{0, k}+1\right)-B_{k+1}(x, y)\right) \\
-B_{k}\left(x+p_{0, k}+1, y+q_{0, k}+1\right)
\end{array}\right),
$$

then $\forall k \in Z^{+}$and $\forall q_{k} \in[0,1]$ we have

$$
\frac{\partial M S E_{k}^{U L}\left(1, q_{k}\right)}{\partial q_{k}}=\widetilde{c}_{k, 1, q} q_{k}+\widetilde{c}_{k, 1} .
$$

$\forall k \in Z^{+}$, denote a stationary point of $\operatorname{MSE}_{k}^{U L}\left(1, q_{k}\right)$ as $\left(1, \tilde{q}_{k}^{1, U L}\right)$. If $\exists k \in Z^{+}$such that $\widetilde{c}_{k, 1, q} \neq 0$ and $-\frac{\widetilde{c}_{k, 1}}{\widetilde{c}_{k, 1, q}} \in[0,1]$, then this stationary point could be the global minimum. For this case, define $\tilde{F}_{k, 1, q}^{U L} \equiv\left\{\left(0,-\frac{\widetilde{c}_{k, 1}}{\widetilde{c}_{k, 1, q}}\right)\right\}$. However, if $\exists k \in Z^{+}$such that $\widetilde{c}_{k, 1, q} \neq 0$ and $-\frac{\widetilde{c}_{k, 1}}{\widetilde{c}_{k, 1, q}} \notin[0,1]$, or $\widetilde{c}_{k, 1, q}=0$, then we do not consider that the global minimum is on the boundary $p_{k}=1 \forall q_{k} \in[0,1]$. For these two cases, define $\widetilde{F}_{k, 1, q}^{U L} \equiv \phi . \forall k \in Z^{+}$and $\forall p_{k} \in[0,1]$,

$$
\begin{aligned}
& \operatorname{MSE}_{k}^{U L}\left(p_{k}, 0\right) \\
& =\left.\frac{1}{N^{2}} \sum_{x=0}^{N-1} \sum_{y=0}^{N-1}\left(\begin{array}{l}
\left(1-p_{k}\right)\left(1-q_{k}\right) B_{k}\left(x+p_{0, k}, y+q_{0, k}\right) \\
+\left(1-p_{k}\right) q_{k} B_{k}\left(x+p_{0, k}+1, y+q_{0, k}\right) \\
+p_{k}\left(1-q_{k}\right) B_{k}\left(x+p_{0, k}, y+q_{0, k}+1\right) \\
+p_{k} q_{k} B_{k}\left(x+p_{0, k}+1, y+q_{0, k}+1\right) \\
-B_{k+1}(x, y)
\end{array}\right)\right|_{q_{k}=0} ^{2} \\
& =\frac{1}{N^{2}} \sum_{x=0}^{N-1} \sum_{y=0}^{N-1}\left(\begin{array}{l}
\left(1-p_{k}\right) B_{k}\left(x+p_{0, k}, y+q_{0, k}\right) \\
+p_{k} B_{k}\left(x+p_{0, k}, y+q_{0, k}+1\right)-B_{k+1}(x, y)
\end{array}\right)^{2} \\
& =\frac{1}{N^{2}} \sum_{x=0}^{N-1} \sum_{y=0}^{N-1}\left(\begin{array}{l}
B_{k}\left(x+p_{0, k}, y+q_{0, k}+1\right) \\
p_{k}\left(\begin{array}{l}
B_{k}\left(x+p_{0, k}, y+q_{0, k}\right) \\
-B_{k}
\end{array}\right) \\
+B_{k}\left(x+p_{0, k}, y+q_{0, k}\right)-B_{k+1}(x, y)
\end{array}\right)^{2}
\end{aligned}
$$

This implies that $\forall k \in Z^{+}$and $\forall p_{k} \in[0,1]$, 


$$
\begin{aligned}
& \frac{\partial M S E_{k}^{U L}\left(p_{k}, 0\right)}{\partial p_{k}} \\
& =\frac{2}{N^{2}} \sum_{x=0}^{N-1} \sum_{y=0}^{N-1}\left(\begin{array}{l}
p_{k}\left(\begin{array}{l}
B_{k}\left(x+p_{0, k}, y+q_{0, k}+1\right) \\
-B_{k}\left(x+p_{0, k}, y+q_{0, k}\right)
\end{array}\right) \\
B_{k}\left(x+p_{0, k}, y+q_{0, k}\right)-B_{k+1}(x, y)
\end{array}\right) \\
& \left.\begin{array}{l}
B_{k}\left(x+p_{0, k}, y+q_{0, k}+1\right) \\
-B_{k}\left(x+p_{0, k}, y+q_{0, k}\right)
\end{array}\right) \\
& =\frac{2}{N^{2}} \sum_{x=0}^{N-1} \sum_{y=0}^{N-1}+\left(\begin{array}{l}
B_{k}\left(x+p_{0, k}, y+q_{0, k}+1\right) \\
-B_{k}\left(x+p_{0, k}, y+q_{0, k}\right)
\end{array}\right) \\
& \left(\begin{array}{l}
B_{k}\left(x+p_{0, k}, y+q_{0, k}\right)-B_{k+1}\left(x+p_{0, k}, y+q_{0, k}+1\right) \\
-B_{k}\left(x+p_{0, k}, y+q_{0, k}\right)
\end{array}\right)
\end{aligned}
$$

$\forall k \in Z^{+}$, denote

$$
\tilde{z}_{k, 0, p} \equiv \frac{2}{N^{2}} \sum_{x=0}^{N-1} \sum_{y=0}^{N-1}\left(\begin{array}{l}
B_{k}\left(x+p_{0, k}, y+q_{0, k}+1\right) \\
-B_{k}\left(x+p_{0, k}, y+q_{0, k}\right)
\end{array}\right)^{2}
$$

and

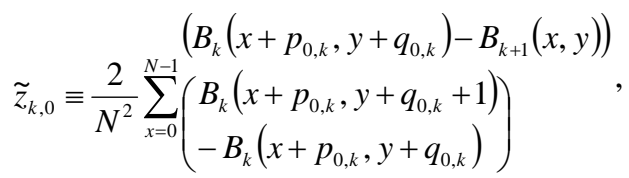

then $\forall k \in Z^{+}$and $\forall p_{k} \in[0,1]$ we have

$$
\frac{\partial M S E_{k}^{U L}\left(p_{k}, 0\right)}{\partial p_{k}}=\widetilde{z}_{k, 0, p} p_{k}+\widetilde{z}_{k, 0} .
$$

$\forall k \in Z^{+}$, denote a stationary point of $\operatorname{MSE}_{k}^{U L}\left(p_{k}, 0\right)$ as $\left(\tilde{p}_{k}^{0, U L}, 0\right)$. If $\exists k \in Z^{+}$such that $\widetilde{z}_{k, 0, p} \neq 0$ and $-\frac{\widetilde{z}_{k, 0}}{\widetilde{z}_{k, 0, p}} \in[0,1]$, then this stationary point could be the global minimum. For this case, define $\widetilde{F}_{k, 0, p}^{U L} \equiv\left\{\left(-\frac{\widetilde{z}_{k, 0}}{\widetilde{z}_{k, 0, p}}, 0\right)\right\}$. However, if $\exists k \in Z^{+}$such that $\widetilde{z}_{k, 0, p} \neq 0$ and $-\frac{\widetilde{z}_{k, 0}}{\widetilde{z}_{k, 0, p}} \notin[0,1]$, or $\tilde{z}_{k, 0, p}=0$, then we do not consider that the global minimum is on the boundary $q_{k}=0 \forall p_{k} \in(0,1]$. For these two cases, define $\tilde{F}_{k, 0, p}^{U L} \equiv \phi$. Lastly, $\forall k \in Z^{+}$and $\forall p_{k} \in[0,1]$,

$$
\begin{aligned}
& \operatorname{MSE}_{k}^{U L}\left(p_{k}, 1\right) \\
& =\left.\frac{1}{N^{2}} \sum_{x=0}^{N-1} \sum_{y=0}^{N-1}\left(\begin{array}{l}
\left(1-p_{k}\right)\left(1-q_{k}\right) B_{k}\left(x+p_{0, k}, y+q_{0, k}\right) \\
+\left(1-p_{k}\right) q_{k} B_{k}\left(x+p_{0, k}+1, y+q_{0, k}\right) \\
+p_{k}\left(1-q_{k}\right) B_{k}\left(x+p_{0, k}, y+q_{0, k}+1\right) \\
+p_{k} q_{k} B_{k}\left(x+p_{0, k}+1, y+q_{0, k}+1\right) \\
-B_{k+1}(x, y)
\end{array}\right)\right|_{q_{k}=1} \\
& =\frac{1}{N^{2}} \sum_{x=0}^{N-1} \sum_{y=0}^{N-1}\left(\begin{array}{l}
\left(1-p_{k}\right) B_{k}\left(x+p_{0, k}+1, y+q_{0, k}\right) \\
+p_{k} B_{k}\left(x+p_{0, k}+1, y+q_{0, k}+1\right)-B_{k+1}(x, y)
\end{array}\right)^{2}
\end{aligned}
$$

$$
=\frac{1}{N^{2}} \sum_{x=0}^{N-1} \sum_{y=0}^{N-1}\left(\begin{array}{l}
p_{k}\left(\begin{array}{l}
B_{k}\left(x+p_{0, k}+1, y+q_{0, k}+1\right) \\
-B_{k}\left(x+p_{0, k}+1, y+q_{0, k}\right)
\end{array}\right) \\
+B_{k}\left(x+p_{0, k}+1, y+q_{0, k}\right)-B_{k+1}(x, y)
\end{array}\right)^{2} .
$$

This implies that $\forall k \in Z^{+}$and $\forall p_{k} \in[0,1]$,

$$
\begin{aligned}
& \frac{\partial M S E_{k}^{U L}\left(p_{k}, 1\right)}{\partial p_{k}} \\
& =\frac{2}{N^{2}} \sum_{x=0}^{N-1} \sum_{y=0}^{N-1}\left(\begin{array}{c}
p_{k}\left(\begin{array}{l}
B_{k}\left(x+p_{0, k}+1, y+q_{0, k}+1\right) \\
-B_{k}\left(x+p_{0, k}+1, y+q_{0, k}\right)
\end{array}\right) \\
+B_{k}\left(x+p_{0, k}+1, y+q_{0, k}\right)-B_{k+1}(x, y)
\end{array}\right) \\
& \left(\begin{array}{l}
B_{k}\left(x+p_{0, k}+1, y+q_{0, k}+1\right) \\
-B_{k}\left(x+p_{0, k}+1, y+q_{0, k}\right)
\end{array}\right) \\
& p_{k}\left(\begin{array}{l}
B_{k}\left(x+p_{0, k}+1, y+q_{0, k}+1\right) \\
-B_{k}\left(x+p_{0, k}+1, y+q_{0, k}\right)
\end{array}\right)^{2} \\
& =\frac{2}{N^{2}} \sum_{x=0}^{N-1} \sum_{y=0}^{N-1}+\left(B_{k}\left(x+p_{0, k}+1, y+q_{0, k}\right)-B_{k+1}(x, y)\right) \\
& \left(\begin{array}{l}
B_{k}\left(x+p_{0, k}+1, y+q_{0, k}+1\right) \\
-B_{k}\left(x+p_{0, k}+1, y+q_{0, k}\right)
\end{array}\right)
\end{aligned}
$$

$\forall k \in Z^{+}$, denote

$$
\tilde{z}_{k, 1, p} \equiv \frac{2}{N^{2}} \sum_{x=0}^{N-1} \sum_{y=0}^{N-1}\left(\begin{array}{l}
B_{k}\left(x+p_{0, k}+1, y+q_{0, k}+1\right) \\
-B_{k}\left(x+p_{0, k}+1, y+q_{0, k}\right)
\end{array}\right)^{2}
$$

and

$$
\widetilde{z}_{k, 1} \equiv \frac{2}{N^{2}} \sum_{x=0}^{N-1} \sum_{y=0}^{N-1}\left(\begin{array}{l}
\left(B_{k}\left(x+p_{0, k}+1, y+q_{0, k}\right)-B_{k+1}(x, y)\right) \\
-B_{k}\left(x+p_{0, k}+1, y+q_{0, k}+1\right)
\end{array}\right),
$$

then $\forall k \in Z^{+}$and $\forall p_{k} \in[0,1]$ we have

$$
\frac{\partial M S E_{k}^{U L}\left(p_{k}, 1\right)}{\partial p_{k}}=\tilde{z}_{k, 1, p} p_{k}+\tilde{z}_{k, 1} .
$$

$\forall k \in Z^{+}$, denote a stationary point of $\operatorname{MSE}_{k}^{U L}\left(p_{k}, 1\right)$ as $\left(\tilde{p}_{k}^{1, U L}, 1\right)$. If $\exists k \in Z^{+}$such that $\tilde{z}_{k, 1, p} \neq 0$ and $-\frac{\widetilde{z}_{k, 1}}{\widetilde{z}_{k, 1, p}} \in[0,1]$, then this stationary point could be the global minimum. For this case, define $\tilde{F}_{k, 1, p}^{U L} \equiv\left\{\left(-\frac{\widetilde{z}_{k, 1}}{\widetilde{Z}_{k, 1, p}}, 1\right)\right\}$. However, if $\exists k \in Z^{+}$such that $\widetilde{z}_{k, 1, p} \neq 0$ and $-\frac{\widetilde{z}_{k, 1}}{\widetilde{z}_{k, 1, p}} \notin[0,1]$, or $\tilde{z}_{k, 1, p}=0$, then we do not consider that the global minimum is on the boundary $q_{k}=1 \forall p_{k} \in[0,1]$. For these two cases, define $\tilde{F}_{k, 1, p}^{U L} \equiv \phi . \forall k \in Z^{+}$, define $F_{k}^{U L} \equiv \tilde{F}_{k, 0, q}^{U L} \cup \tilde{F}_{k, 1, q}^{U L} \cup \tilde{F}_{k, 0, p}^{U L} \cup \tilde{F}_{k, 1, p}^{U L} \cup\{(0,0)\}$.

Similarly, $\forall k \in Z^{+}$, denote the set of motion vectors corresponding to the stationary points of $\operatorname{MSE}_{k}^{U R}\left(p_{k}, q_{k}\right)$, $\operatorname{MSE}_{k}^{L L}\left(p_{k}, q_{k}\right)$ and $\operatorname{MSE}_{k}^{L R}\left(p_{k}, q_{k}\right)$ (including the point $(0,0))$ as $F_{k}^{U R}, F_{k}^{L L}$ and $F_{k}^{L R}$, respectively. The algorithm for finding the globally optimal motion vector can be summarized as follow:

Algorithm 
Step 1: Implement an existing full integer pixel search algorithm so that $\left(p_{0, k}, q_{0, k}\right)$ is obtained $\forall k \in Z^{+}$.

Step 2: $\forall k \in Z^{+}$, evaluate $F_{k}^{U L}, F_{k}^{U R}, F_{k}^{L L}$ and $F_{k}^{L R}$.

Step 3: $\forall k \in Z^{+}$, evaluate

$$
\left(p_{k}^{*}, q_{k}^{*}\right) \equiv \arg \left\{\begin{array}{l}
\arg \left\{\min _{\left(p_{k}, q_{k}\right) \in F_{k}^{U L}} \operatorname{MSE}_{k}^{U L}\left(p_{k}, q_{k}\right)\right\}, \\
\arg \left\{\min _{\left(p_{k}, q_{k}\right) \in F_{k}^{U R}} M S E_{k}^{U R}\left(p_{k}, q_{k}\right)\right\}, \\
\arg \left\{\min _{\left(p_{k}, q_{k}\right) \in F_{k}^{L L}} M S E_{k}^{L L}\left(p_{k}, q_{k}\right)\right\}, \\
\arg \left\{\min _{\left(p_{k}, q_{k}\right) \in F_{k}^{L R}} M S E_{k}^{L R}\left(p_{k}, q_{k}\right)\right\}
\end{array}\right\} .
$$

$\forall k \in Z^{+}$, take $\left(p_{k}^{*}, q_{k}^{*}\right)$ as the globally optimal motion vector of $B_{k}$.

Since the global minimum of the mean square error is not necessarily located at rational pixel locations, while the full integer pixel search, full half pixel search and full quarter pixel search algorithms only evaluate at rational pixel locations, the mean square errors based on these conventional methods are very large and these conventional methods are very ineffective. On the other hand, our proposed method guarantee to find the motion vector that globally minimizes the mean square error no matter the motion vector is located at either rational pixel locations or irrational pixel locations. Hence, our proposed method is more effective that conventional methods. Besides, as integer pixel locations, half pixel locations and quarter pixel locations are particular locations represented by our proposed model, the mean square error based on our proposed method is guaranteed to be lower than that based on these conventional methods.

The computational effort of our proposed algorithm can be analyzed as follows. As the orders of the polynomials in (1), (2) and (3) are 5, 4 and 2, respectively, $0 \leq M_{k}^{U L} \leq 5$ $\forall k \in Z^{+}$. Hence, $\forall k \in Z^{+}$, if $M_{k}^{U L} \geq 1$, then the maximum evaluation points of our proposed method are less than or equal to 21. $\forall k \in Z^{+}$, if $M_{k}^{U L}=0$, as the maximum number of points in $F_{k}^{U L}$ is 5 , the maximum evaluation points of our proposed method are less than or equal to 17 . For full quarter pixel search algorithms, there are 25 evaluation points. Hence, the total number of evaluation points of our proposed method is lower than that of full quarter pixel search algorithms. As conventional block matched motion estimation algorithms evaluate block matching errors from coarse pixel locations to fine pixel locations, the computational efforts grow exponentially as the pixel precisions get finer and finer. From this point of view, the conventional methods are very inefficient. On the other hand, our proposed method does not require searching from the coarse pixel locations to the fine pixel locations. Our proposed method is more efficient than the conventional methods particularly when the required pixel precision is higher than or equal to the quarter pixel precisions.

\section{SimULATION RESULTS}

In order to have complete investigations, video sequences with fast motion, medium motion and slow motion are studied. The video sequences, Foreman, Coastguard and Container [7], are, respectively, the most common fast motion, medium motion and slow motion video sequences. Hence, motion estimations are performed to these video sequences. Except the first frame of these video sequences, the mean square errors of all the frames of these video sequences are evaluated. Each current frame takes its immediate predecessor as the reference frame. The sizes of the marco blocks are chosen as $8 \times 8$ and $16 \times 16$ and the sizes of the search windows are chosen as 32 and 40 , which are the most common block sizes and window sizes used in international standards. The comparisons are made with the full integer pixel search algorithm, the full half pixel search algorithm and the full quarter pixel search algorithm.

The mean square error performances of our proposed method, the full integer pixel search algorithm, the full half pixel search algorithm and the full quarter pixel search algorithm with the size of the marco blocks $8 \times 8$ and the size of the search windows 32 applied to the video sequences Coastguard, Container and Foreman are shown in Figure 1a, Figure 1b, and Figure 1c, respectively. It can be seen from the Figure 1 that the improvements on the average mean square errors of the full half pixel search algorithm, the full quarter pixel search algorithm and our proposed method over the full integer search algorithm for the video sequences Coastguard are $1.4894 \times 10^{-4}$, $2.2242 \times 10^{-4}$ and $2.7294 \times 10^{-4}$, respectively, which correspond to $17.8531 \%, 28.8039 \%$ and $37.5835 \%$, respectively, that for the video sequences Container are $1.4406 \times 10^{-6}, 3.6476 \times 10^{-6}$ and $2.0374 \times 10^{-5}$, respectively, which correspond to $1.0115 \%, 4.4170 \%$ and $32.3070 \%$, respectively, and that for the video sequences Foreman are $1.5788 \times 10^{-4}, 2.2863 \times 10^{-4}$ and $2.5897 \times 10^{-4}$, respectively, which correspond to $24.7674 \%$, 39.1977\% and 46.4394\% , respectively. Similar results are obtained for different size of marco blocks and different size of the search windows. Figure 2 shows the improvements on the average mean square errors of various algorithms with the size of the marco blocks $16 \times 16$ and the size of the search windows 40 applied to the same set of video sequences. The improvements on the average mean square errors of the full half pixel search algorithm, the full quarter pixel search algorithm and our proposed method over the full integer search algorithm for the video sequences Coastguard are $1.7838 \times 10^{-4}, 2.5650 \times 10^{-4}$ and $3.0888 \times 10^{-4}$, respectively, which correspond to $18.4666 \%, 27.6579 \%$ and $34.6995 \%$, respectively, that for the video sequences Container are $1.8757 \times 10^{-6}$, $2.5444 \times 10^{-6}$ and $1.8031 \times 10^{-5}$, respectively, which correspond to $0.7710 \%, 1.5106 \%$ and $26.9046 \%$, respectively, and that for the video sequences Foreman are $2.1073 \times 10^{-4}, 2.9528 \times 10^{-4}$ and $3.3051 \times 10^{-4}$, respectively, which correspond to $21.6021 \%$, 34.2148\% and $40.4725 \%$, respectively. From the above computer numerical simulations, it can be concluded that the mean square error performances of our proposed method are always better than the full integer pixel search algorithm, the full half pixel search algorithm and the full quarter pixel search algorithm for all of the above three video sequences. In particular, for slow motion video sequences, such as the video sequence Container, our proposed 
method significantly outperforms the full integer pixel search algorithm, the full half pixel search algorithm and the full quarter pixel search algorithm. This is because the globally optimal motion vectors for these slow motion video sequences are very close to the origin and far from the half pixel locations and the quarter pixel locations. In this case, the full half pixel search algorithm and the full quarter pixel search algorithm would not yield very significant improvements over the full integer pixel search algorithm. On the other hand, our proposed method could solve the globally optimal motion vectors with very high pixel precisions and hence yields very significant improvements.
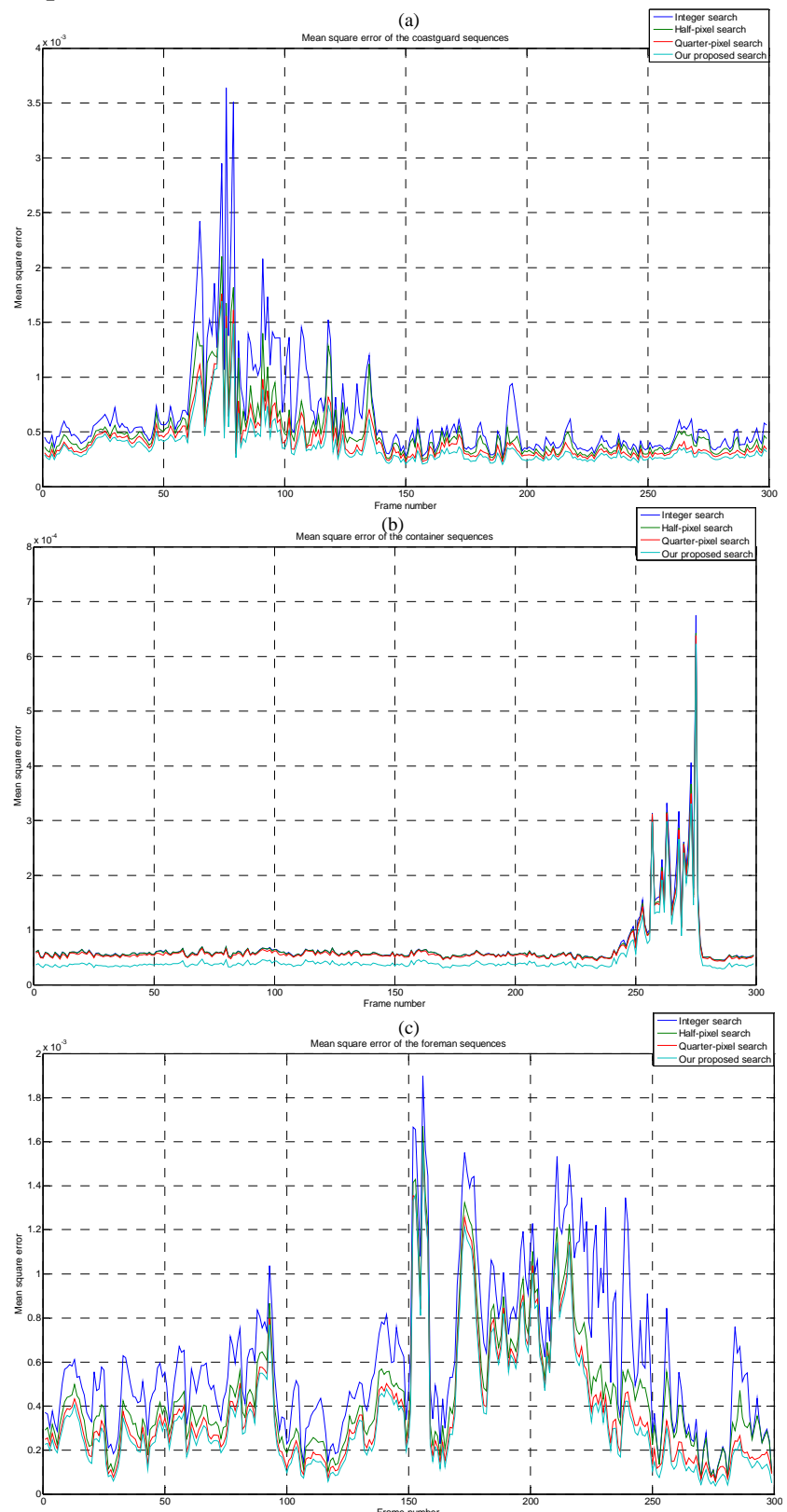

Figure 1. The mean square error performances of our proposed method, the full integer pixel search algorithm, the full half pixel search algorithm and the full quarter pixel search algorithm with the size of the marco blocks $8 \times 8$ and the size of the search windows 32 applied to the video sequences Coastguard, Container and Foreman.
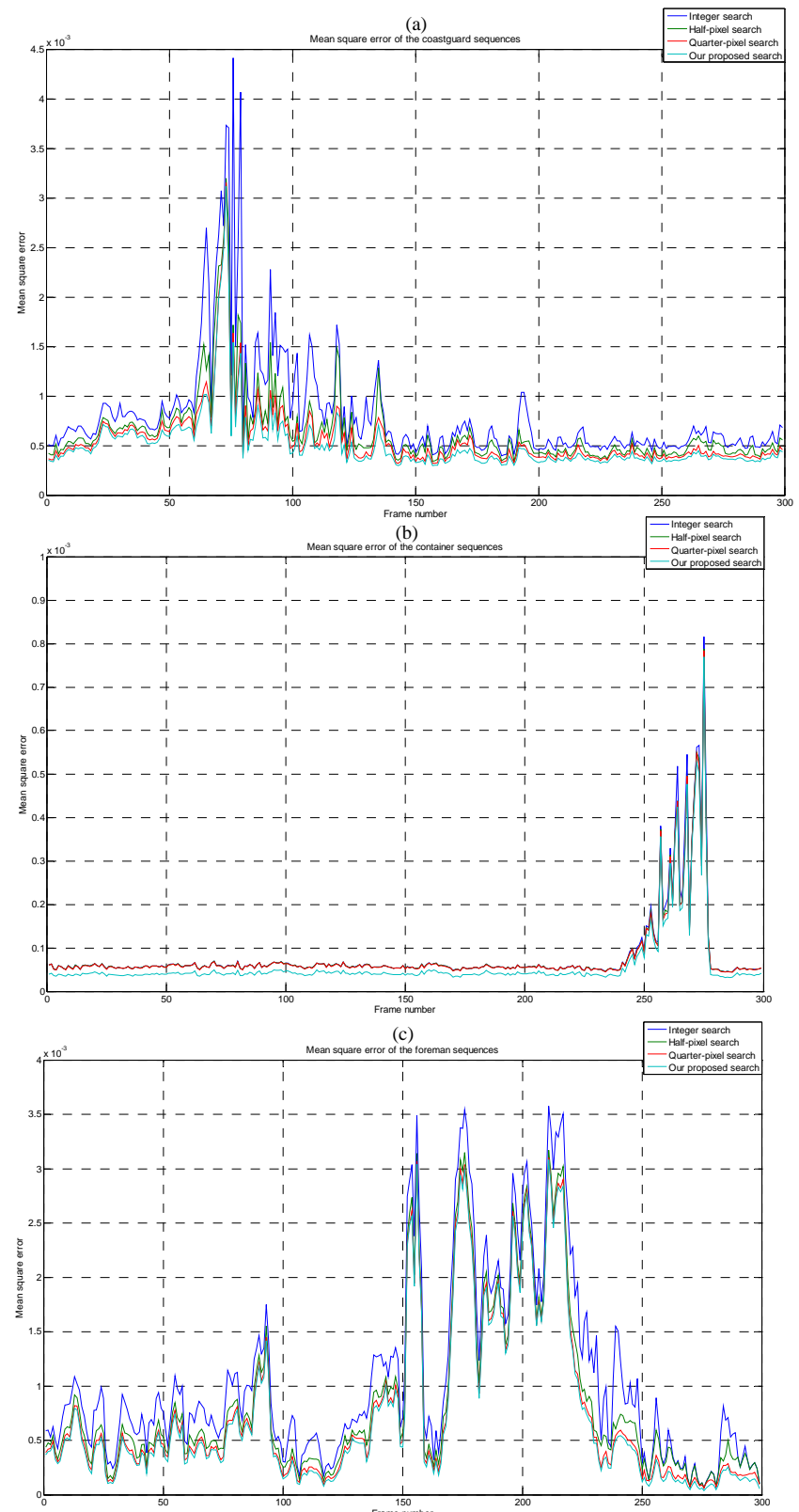

Figure 2. The mean square error performances of our proposed method, the full integer pixel search algorithm, the full half pixel search algorithm and the full quarter pixel search algorithm with the size of the marco

blocks $16 \times 16$ and the size of the search windows 40 applied to the video sequences Coastguard, Container and Foreman.

\section{CONCLUSION}

A nonlinear block matched motion model is proposed in this paper. The motion vector which globally minimizes the mean square error is solved analytically. Hence, a motion vector with an infinite pixel precision is obtained at a single step. As integer pixel locations, half pixel locations and quarter pixel locations are particular locations represented by our proposed model, the mean square error based on our proposed method is guaranteed to be lower than that based on these conventional methods. Also, as our proposed method does not require searching from coarse pixel locations to fine pixel locations, our proposed method is more efficient than conventional methods particularly when the required pixel precision is higher than or equal to the quarter pixel precisions. 


\section{ACKNOWLEDGEMENT}

The work obtained in this paper was supported by a research grant from the Center for Multimedia Signal Processing (under project BB9D), The Hong Kong Polytechnic University.

\section{REFERENCES}

[1] Se Young Chun and Jeffrey A. Fessler, “A simple regularizer for B-spline nonrigid image registration that encourages local invertibility," IEEE Journal of Selected Topics in Signal Processing, vol. 3, no. 1, pp. 159-169, 2009.

[2] I-Chen Lin, Jeng-Sheng Yeh and Ming Ouhyoung, "Extracting 3D facial animation parameters from multiview video clips," IEEE Computer Graphics and Applications, vol. 22, no. 6, pp. 72-80, 2002.

[3] Avishek Saha, Jayanta Mukherjee and Shamik Sural, "New pixeldecimation patterns for block matching in motion estimation," Signal Processing: Image Communication, vol. 23, no. 10, pp. 725-738, 2008.

[4] Xiaoming Li and Cesar Gonzales, “A locally quadratic model of the motion estimation error criterion function and its application to subpixel interpolations," IEEE Transactions on Circuits and Systems for Video Technology, vol. 6, no. 1, pp. 118-122, 1996.

[5] Cheng Du, Yun He and Junli Zheng, "PPHPS: A parabolic prediction-based, fast half-pixel search algorithm for very low bitrate moving-picture coding," IEEE Transactions on Circuits and Systems for Video Technology, vol. 13, no. 6, pp. 514-518, 2003.

[6] Yun-Gu Lee, Jae Hun Lee and Jong Beom Ra, "Fast half-pixel motion estimation based on directional search and a linear model," Proceedings of SPIE Visual Communications and Image Processing, vol. 5150, pp. 1513-1520, 2003.

[7] [Online]. Available:

http://www.cipr.rpi.edu/resource/sequences/sif.html 